УДК 624.131.52

\title{
А.В. Пилягин
}

Чебоксарский политехнический институт, филиал Московского государственного машиностроительного университета (МАМИ), Чебоксары, Россия

\section{НАПРЯЖЕННО-ДЕФОРМИРОВАННОЕ СОСТОЯНИЕ ОСНОВАНИЙ КРУГЛЫХ ФУНДАМЕНТОВ ПРИ РАЗЛИЧНЫХ СХЕМАХ ЗАГРУЖЕНИЯ}

Приводятся аналитические решения по определению вертикальных напряжений и перемещений (осадок) фундаментов круглой формы при различных схемах их загружения

Ключевые слова: напряжение, осадка, нагрузка, фундамент [1, 2].

\author{
A.V. Pilyagin \\ Cheboksary Polytechnic Institute, Cheboksary, Russian Federation

\section{STRESS-STRAIN STATE OF THE BASES OF THE ROUND} \\ FOUNDATIONS WITH DIFFERENT SCHEMES OF LOADING
}

Provides analytical decision on determination of the vertical stresses and displacements (sludge) foundations of the round form with different schemes of their loading.

Keywords: stress, settlement, load, foundation.

В соответствии с решением Буссинеска вертикальное напряжение в основании при действии на него поверхности единичной сосредоточенной силы $P$ вычисляется по формуле

$$
\begin{gathered}
\sigma_{z}=\frac{3 P}{2 \pi} \frac{z^{3}}{R^{5}}, \\
R=\sqrt{x^{2}+y^{2}+z^{2}},
\end{gathered}
$$

где $x, y, z$ - координаты рассматриваемой точки основания.

На рис. 1 рассматриваются три схемы приложения нагрузки по круговой площадке. 


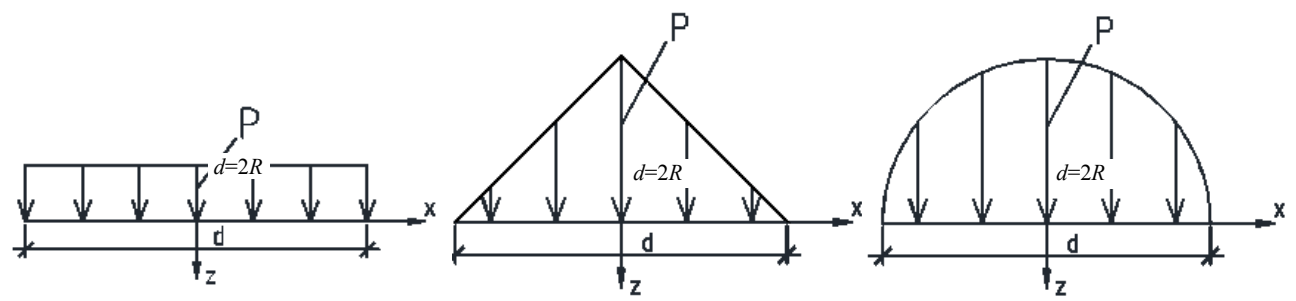

Рис. 1. Схемы загружения: $a$ - нагрузка равномерно-распределенная; $\sigma$ - нагрузка в виде конуса $P_{i}=P(1-x / R) ;$; - изменение нагрузки по параболе $P_{i}=P\left(1-x^{2} / R^{2}\right)$

Для вычисления вертикальных напряжений по центральной оси необходимо выражение (1) проинтегрировать по площади заглубления, т.е. вычислить

$$
\begin{aligned}
& \sigma_{z}=\frac{3 P \cdot z^{3}}{2 \pi} \int_{0}^{2 \pi} d \varphi \int_{0}^{R} \frac{x \cdot d x}{\left(x^{2}+z^{2}\right)^{5 / 2}}=\frac{3 P \cdot z^{3}}{2 \pi}\left|\frac{1}{3 z^{3}}-\frac{1}{3\left(\sqrt{R^{2}+z^{2}}\right)^{3}}\right|= \\
& =P\left[1-\frac{1}{\left(1+\frac{R^{2}}{z^{2}}\right)^{3 / 2}}\right] .
\end{aligned}
$$

При приложении нагрузки, изменяющейся по закону конуса (рис. $1, \sigma)$, т.е. $P_{i}=P(1-x / R)$, вертикальные напряжения можно вычислить по формуле

$$
\begin{aligned}
& \sigma_{z}=\frac{3 P \cdot z^{3}}{2 \pi} \int_{0}^{2 \pi} d \varphi \int_{0}^{R} \frac{\left(1-\frac{x}{R}\right) \cdot x \cdot d x}{\left(x^{2}+z^{2}\right)^{5 / 2}}=\frac{3 P \cdot z^{3}}{2 \pi}\left|\frac{1}{3 z^{3}}-\frac{1}{3 z^{2} \sqrt{R^{2}+z^{2}}}\right|= \\
& =P\left[1-\frac{z}{\sqrt{R^{2}+z^{2}}}\right] .
\end{aligned}
$$

При $z=0$, значение $\sigma_{z}=P$.

При приложении нагрузки, изменяющейся по закону параболы (рис. 1,8 ), вертикальные напряжения по центральной оси могут быть вычислены по выражениям

$$
\sigma_{z}=\frac{3 P \cdot z}{2 \pi} \int_{0}^{2 \pi} d \varphi \int_{0}^{R}\left(1-\frac{x^{2}}{R^{2}}\right) \frac{x \cdot d x}{\left(x^{2}+z^{2}\right)^{5 / 2}}=\frac{P}{R^{2}}\left|\frac{z^{3}}{\sqrt{R^{2}+z^{2}}}-2 z^{2}+R^{2}\right| .
$$


При $z=0$, значение $\sigma_{z}=P$.

В соответствии с решением Буссинеска вертикальные перемещения точек основания от действия единичной сосредоточенной силы

$$
S=\frac{(1+\mu) P}{2 \pi \cdot E}\left[\frac{z^{2}}{R^{3}}+2(1-\mu) \frac{1}{R}\right],
$$

где $\mu$ - коэффициент Пуассона грунта.

Для определения осадки токи основания по центральной вертикали загруженной круговой площадки необходимо проинтегрировать выражение (5) по площади круга, т.е. решить интеграл:

$$
\begin{aligned}
& S=\frac{(1+\mu) P \cdot z^{2}}{2 \pi \cdot E} \int_{0}^{2 \pi} \int_{0}^{R} \frac{x \cdot d x \cdot d \varphi}{\left(\sqrt{x^{2}+z^{2}}\right)^{3}}+\frac{(1-\mu)^{2} P^{2 \pi}}{\pi \cdot E} \int_{0}^{R} \int_{0}^{R} \frac{x \cdot d x \cdot d \varphi}{\sqrt{x^{2}+z^{2}}}= \\
& =\frac{(1+\mu) P}{E}\left[z-\frac{z^{2}}{\sqrt{R^{2}+z^{2}}}\right]+\frac{(1-\mu)^{2} 2 P}{E}\left(\sqrt{R^{2}+z^{2}}-z\right) .
\end{aligned}
$$

Или при $z=0$, получим известное решение

$$
S=\frac{(1-\mu)^{2} P \cdot d}{E} .
$$

При приложении нагрузки в виде конуса величина осадки может быть вычислена по формуле

$$
\begin{gathered}
S=\frac{\left(1-\mu^{2}\right) P}{\pi \cdot E} \int_{0}^{2 \pi} d \varphi \int_{0}^{R} \frac{\left(1-\frac{x}{R}\right) x \cdot d x}{\left(x^{2}+z^{2}\right)^{1 / 2}}+\frac{(1+\mu) P \cdot z^{2}}{2 \pi \cdot E} \int_{0}^{2 \pi} d \varphi \int_{0}^{R} \frac{\left(1-\frac{x}{R}\right) x \cdot d x}{\left(x^{2}+z^{2}\right)^{3 / 2}}= \\
=\frac{\left(1-\mu^{2}\right) P}{E}\left[\sqrt{R^{2}+z^{2}}+z^{2} / R \cdot \ln \left(R+\sqrt{R^{2}+z^{2}}\right)-1 / R \cdot z^{2} \ln (z)+2 z\right]+ \\
+\frac{z^{2}(1+\mu) P}{E \cdot R}\left[\ln (z)+R-\ln \left(R+\sqrt{R^{2}+z^{2}}\right)\right] .
\end{gathered}
$$

Или при $z=0$

$$
S=\frac{\left(1-\mu^{2}\right) P \cdot d}{2 E} .
$$


При приложении нагрузки, изменяющейся по закону параболы, величина осадки на центральной вертикали может быть вычислена по формуле

$$
\begin{gathered}
S=\frac{\left(1-\mu^{2}\right) P}{\pi \cdot E} \int_{0}^{2 \pi} d \varphi \int_{0}^{R} \frac{\left(1-\frac{x^{2}}{R^{2}}\right) x \cdot d x}{\left(x^{2}+z^{2}\right)^{1 / 2}}+\frac{(1+\mu) P \cdot z^{2}}{2 \pi \cdot E} \int_{0}^{2 \pi} d \varphi \int_{0}^{R} \frac{\left(1-\frac{x^{2}}{R^{2}}\right) x \cdot d x}{\left(x^{2}+z^{2}\right)^{3 / 2}}= \\
=\frac{\left(1-\mu^{2}\right) 2 P}{E}\left[\frac{2}{3} \frac{\left(R^{2}+z^{2}\right)^{3 / 2}}{R^{2}}-\frac{1}{3} z \cdot \frac{2 z^{2}+3 R^{2}}{R^{2}}\right]+ \\
+\frac{P(1+\mu) \cdot z^{2}}{E \cdot R^{2}}\left[\frac{R^{2}+2 z^{2}}{z}-\sqrt{R^{2}+z^{2}}\right] .
\end{gathered}
$$

Или при $z=0$

$$
S=\frac{2}{3} \frac{\left(1-\mu^{2}\right) P \cdot d}{E}
$$

\section{Библиографический список}

1. Пилягин А.В. Об определении модуля общей деформации грунтов по данным полевых испытаний // ОФМГ. - 2013. - № 2. - С. 25-29.

2. Пилягин А.В. Напряженно-деформированное состояние оснований фундаментов зданий и сооружений. - Чебоксары: Изд-во Чебоксар. политехн. ин-та, 2010. - 262 с.

\section{References}

1. Pilyagin A.V. Ob opredelenii modulya obshchej deformatsii gruntov po dannym polevykh ispytanij [On the determination of total soil deformation modulus according to field trials]. Osnovaniya, fundamenty $i$ mehanika gruntov, 2013, no. 2, pp.25-29.

2. Pilyagin A.V. Napryazhenno-deformirovannoe sostoyanie osnovanij funda-mentov zdanij i sooruzhenij [Stress-strain state of foundations of buildings and structures]. Cheboksary politekhnichesciy institut, 2010. $262 \mathrm{~s}$. 


\section{Об авторах}

Пилягин Алексей Васильевич (Чебоксары, Россия) - доктор технических наук, заведующий кафедрой Чебоксарского политехнического института, филиала Московского государственного машиностроительного университета (МАМИ); e-mail: pilyagin.alexei@yandex.ru

\section{About the authors}

Pilyagin Aleksey Vasilevich (Togliatti, Russian Federation) - Doctor of Technical Sciences, Head of Cheboksary Polytechnic th Institute, a branch of the Moscow State University of machine-building (MAMI); e-mail: pilyagin.alexei@yandex.ru

Получено 31.03.2014 